\title{
Self-assembly of active core corona particles into highly ordered and self-healing structures
}

\author{
Yunfei Du, Huijun Jiang, and Zhonghuai Hou* \\ Hefei National Laboratory for Physical Sciences at Microscales and Department of Chemical Physics, \\ iChEM, University of Science and Technology of China, Hefei, Anhui 230026, Chind
}

\begin{abstract}
Formation of highly ordered structures usually needs to overcome a high free-energy barrier that is greatly beyond the ability of thermodynamic fluctuation, such that the system would be easily trapped into a state with many defects and the annealing process of which often occurs on unreachable long time-scales. Here we report a fascinating example theoretically that active core corona particles can successfully self-assemble into a large-scaled and highly ordered stripe or trimer lattice, which is hardly achieved in a non-driven equilibrium system. Besides, such an activity-induced ordered structure shows an interesting self-healing feature of defects. In addition, there exists an optimal level of activity that most favorably enhance the formation of ordered self-assembly structures. Since core corona particles act as important units for self-assembly in real practice, we believe our study opens a new design-strategy for highly ordered materials.
\end{abstract}

\section{INTRODUCTION}

Significant advancement has been made in selfassembly of colloidal particles in recent decades [1, 2]. It is now possible to manipulate various types of colloidal particles to fabricate them into highly ordered functional structures 3, 4, widely exploited in various fields such as in photonic [5, 6], phononic [7, 8] or lithographic applications 9 . A popular choice recently for self-assembly unit is the so-called core corona particle, which consists of an inside core (usually metallic nanoparticle) surrounded by ambient soft corona (e.g., polymer chains or microgels) to prevent the aggregation of cores 10]. The interaction between two core corona particles is characterized by two repulsive length scales, related to the hard and soft repulsion, respectively. It is reported that such interaction can result in phases with non-trivial symmetries which include loose- and closepacked hexagonal lattice, monomer, dimer, and trimer fluids, stripe and labyrinthine phases, honeycomb lattice, etc 11, 12 and even quasicrystals with unexpectedly high symmetry[13].

Note that the highly ordered equilibrium phase of the system mentioned above correspond to the state with lowest free energy that is thermodynamically most stable for given parameters. Such ordered structures may have great importance in material science, e.g., being used as templates for lithography nanomanufacturing which requires zero-defect patterns with even molecular level tolerance 14. In a real process of self-assembly in experiment, however, one generally starts from random initial conditions and the system would easily get trapped into a metastable state that is not fully ordered but with many defects. There typically exists a high free energy barrier between this metastable state and the equilibrium ordered one, which is hardly overcome by thermodynamic fluctuations [15], such that the system would stay at the

\footnotetext{
*hzhlj@ustc.edu.cn
}

metastable state with defects for prohibitively long timescales [16, 17. Therefore, how to obtain a highly ordered structure with low fraction of defects as much as possible becomes a challenging problem in the study of selfassembly.

Recently, dynamics of self-propelled active colloids which absorb energy from ambient environment and push the system to a state far from equilibrium, has gained extensive research attentions. A wealth of remarkable collective behaviors have been reported both experimentally and theoretically, including phase separation[18 20], active turbulence[21], active swarming[22], etc. Very recently, a few important works have noticed the perspective of using active particle to alter the properties of colloidal aggregates [23. For example, A. Cacciuto et.al used a collection of triangular colloidal blocks with activity to achieve the self-assembly of capsid-like structures 24. In a subsequent work, they found that activity can also remarkably enhance the self-assembly process of triblock Janus colloids into kagome lattice[25. These works suggested that it is possible to design highly ordered structures by introducing activity into the assembly unit.

Here in the present work, we report theoretically an example that active core corona particles can be successfully self-assembled into a large scaled and highly ordered structure. In the absence of activity, the extruding between core corona particles at dense systems strongly suppress the local arrangements, thus the system would be trapped into metastable states with much defects as mentioned above. We find, however, if exerting an active force for each particle, the system would form a highly ordered stripe pattern or trimer lattice. In particular, such an activity-induced ordered structure owns a selfhealing feature such that the system can maintain a very low defect ratio as long as the activity exists. Besides, we also find that activity has a two-fold effect: while a moderate activity can facilitate the emergence of ordered structure, a strong activity could destroy the order. Therefore, it is convenient to tune the system order by 
(a)

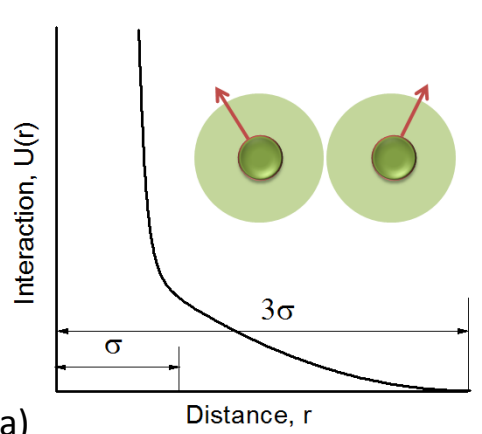

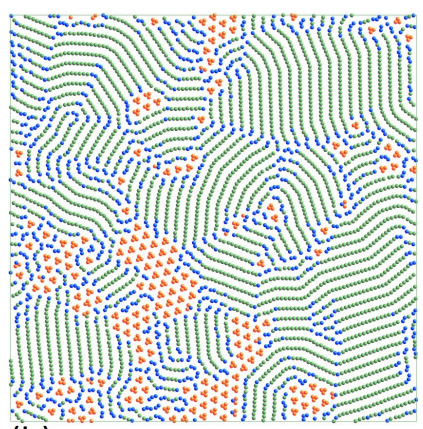

(b)

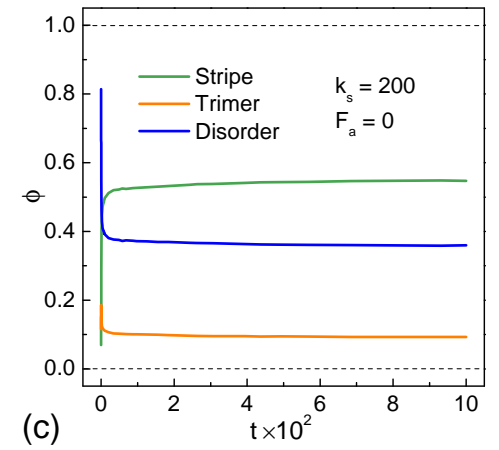

(c)

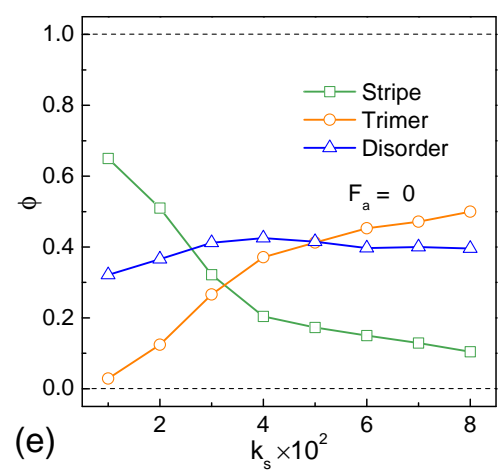

Figure 1. Model setting and the characteristics of a passive system. (a) Equivalent interaction of a pair of active core corona particles. The core colored in dark green is modeled as unpenetrable colloid which is propelled by an active force $F_{a}$ depicted by red arrow. Note $F_{a}=0$ for (b)-(e), corresponding a passive case. The interaction between the ambient coronas colored in light green is modeled by a simple spring, which the strength of it is $k_{s}$. (b) Stable spatial configuration of the system at $k_{s}=200$. Note that the coronas are not drawn out for simplicity. Such a configuration consists of three typical phases, namely stripe phase colored in green, trimer phase colored in yellow and disorder phase colored in blue. (c) Fractions of three phases $\phi_{i}(i=S, T, D$ corresponding to stripe, trimer and disorder, respectively) over time. (d) Equilibrium phase diagram. The phase boundary between stripe phase and trimer phase is at $k_{s}=650$ approximately. (e) Stable values of the fractions $\phi_{i}$ as a function of $k_{s}$.

simply changing the strength of activity. Our results may predict a new general routine to achieve high-ordered and self-healing structures by simply introducing active force to self-assembly system.

\section{RESULT AND DISCUSSION}

We perform two-dimensional overdamped Langevin dynamics simulation of 4096 active core corona particles $(\mathrm{ACCP})$ in a periodic square box. In Figure 1(a), a schematic diagram of a pair of ACCPs is shown. The cores with diameter $\sigma$ model the unpenetrable colloidal spheres described by Weeks-Chandler-Andersen (WCA)potential. The interaction between the ambient coronas, in consideration of the essential entropy-elastic property of polymers or microgels [15, 26, is described by a simple spring potential with equilibrium distance $r_{c}=3 \sigma$ and is also cutoff at $r_{c}$. The interaction as a function of distance between two ACCPs is depicted by the curve in Figure 1(a), which consists of a soft-repulsion part in longdistance range between coronas and a strong-repulsion part in short-distance range between cores. Additionally, each core of ACCP is propelled by an active force $F_{a}$ (shown by the red arrow in Figure 1(a)) with an orientation undergoing random Brownian rotation. The square box length is set to $100 \sigma$, corresponding to a number density $c \simeq 0.41$. In the present work, the strength of $F_{a}$ and the strength of spring potential $k_{s}$ are used as free parameters, and all parameters are dimensionless. A more thorough description of simulation method is provided in Supporting Information S1.

\section{A. Passive case}

We first consider the passive case with $F_{a}=0$. The strength of spring potential $k_{s}$ is fixed to be 200 if not otherwise stated. Figure 1(b) shows the stationary pattern of the system after a long enough time, starting from a random initial condition. Note that the coronas are not drawn out for simplicity. Typically, there exist three kinds of local structure phases, i.e., stripe phase (green), trimer phase (yellow) and disorder phase (blue). To identify which phase a particle belongs to, we can evaluate the angle $\theta\left(0<\theta<180^{\circ}\right)$ of it with its two nearest 
neighbors. A particle is in the stripe phase if $\theta>160^{\circ}$, trimer phase if $50^{\circ}<\theta<70^{\circ}$ and disorder phase otherwise. As can be seen from the figure, a large portion of the pattern are in ordered stripe and trimer phases. In Figure 1. (c), the fractions $\phi_{i}(i=S, T, D$ corresponding to stripe, trimer and disorder, respectively) of each phase as functions of time $t$ are presented, starting from a disordered state with $\phi_{D} \simeq 0.8$. Clearly, the system relaxes very fast to the stationary state wherein the values of $\phi_{i}$ reach stable values, say $\phi_{S} \simeq 0.5, \phi_{T} \simeq 0.1$ and $\phi_{D} \simeq 0.4$. Such a mixed state with coexistence of stripe, trimer and disorder phases is very stable (as can be seen from the time dependencies of $\phi_{i}$ ) and we have not observed any obvious changes to the configuration after very long simulation time. That means thermal fluctuations in such a crowded system play an insignificant role to the dynamic process, in consistent with previous experimental observations [15, 27.

One should note that the mixed state shown in Figure 1(b) is actually a metastable state of the system, rather than the equilibrium state with the lowest free energy. The equilibrium phase diagram in terms of $k_{s}$ (see methods in ref[28 30]) of the system is shown in Figure 1 (d). As one can see, the system shows two distinct equilibrium phases, namely stripe and trimer, with the variation of control parameter $k_{s}$ and the phase boundary is at $k_{s} \simeq 650$. Therefore, the most stable state of the system with lowest free energy for $k_{s}=200$ should be a ordered stripe phase rather than the mixed one in Figure 1(b). The reason that one observes a mixed-phase state which is metastable rather than the equilibrium stripe phase is that there might be a rather high free energy barrier between them, and the attraction basin of the metastable one is much larger than that of the equilibrium one. Starting from a random initial condition, it is highly probable that the system will get trapped into the local minimum of the free energy landscape corresponding to the metastable state, and the high barrier makes it very difficult for the system to jump into the equilibrium state. It is also interesting to note that both the stripe and trimer structures are anisotropic, while the interaction between the particles are purely isotropic. Indeed, this reflects a kind of symmetry breaking. In such a dense system, if particles are equally spaced from each other, the system would obtain a high energetic cost since the corona of each particle overlaps with all its nearest neighbors. If the system chooses a stripe or trimer configuration, however, despite of close packing with two nearest particles, a particle would keep away from other ones as much as possible and finally minimize the energetic $\operatorname{cost} 31$.

As indicated by Figure1(d), the equilibrium state of the system would change from stripe to trimer with increment of $k_{s}$. For $k_{s}=200$ as shown in Figure 1(c), the observed metastable state has more stripe particles than trimer particles, $\phi_{S}>\phi_{T}$. In Figure 1(e), we show how the stable values of the fractions $\phi_{i}$ change with $k_{s}$. With increasing $k_{s}, \phi_{T}\left(\phi_{S}\right)$ increases (decreases) monotoni- cally as expected, while the fraction of disordered phase does not change much. The observed patterns all look similar to that shown in Figure 11(b), except that the fractions of different phases are different. Therefore, starting from a random initial condition, the system would finally get trapped into a metastable mixed-phase state no matter what the value $k_{s}$ is. Indeed, this makes it hard to obtain very ordered structure in practical self-assembly processes and it is demanding to find a way to overcome this difficulty.

\section{B. Active case}

We now put the system (still $k_{s}=200$ ) far from equilibrium by adding a nonzero active force to each particle, starting from similar random initial condition as for the passive case. In Figure2(a)-(d), we give typical snapshots of the system during the relaxation process for an intermediate level of active force $F_{a}=30$. Very interestingly, the final state shows a perfect stripe phase (Figure2(d)) which is in sharp contrast to the metastable mixed-phase shown in Figure 1(b) for $F_{a}=0$. This stripe phase looks the same as the equilibrium one for $k_{s}=200$ shown in Figure1(d). Therefore, the system successfully self-assemble into the demanded highly-ordered state by making the particles active.

The transition from the initial disordered state (a) to the final ordered state (d) consists of three main steps. In the first step from (a) to (b), one can see that most of the disorder phase transform into more ordered stripe or trimer phase, and the remaining trimer-phase particles aggregate together to form a small island surrounded by stripe phase. This trimer island will survive for some time, and since stripe is more stable than trimer according to the equilibrium phase diagram (Figure. 11(d)) for $k_{s}=200$, in the second step from (b) to (c), the trimer island will finally be eliminated and the system changes into a state dominated overwhelmingly by stripe phase. Such a stripe-dominated state still contains some disorder defects, and in the third step from (c) to (d), the system finally transforms into a nearly perfect stripe phase with no apparent defects by local rearrangement and relaxation (see Movie 1). Accordingly, the time dependence of $\phi_{S}$ is shown in Figure/2(e). As can be seen, $\phi_{S}$ increases fastly at the very beginning to a relatively high value about 0.78 (see the yellow cross in Figure2(e)), corresponding to the first step. Then $\phi_{S}$ keeps at a plateau $\phi_{S} \simeq 0.88$ for a quite long period of time. Clearly, the plateau is related to the metastable trimer island which will survive for some time before losing stability. The final slow increasing of $\phi_{S}$ to 1.0 corresponds to the third relaxation step. Note that the life time of the trimer island randomly changes from simulation run to run, but the trimer island can always be observed during the process.

The above observations clearly demonstrate that particle activity can induce very exciting behavior that is 

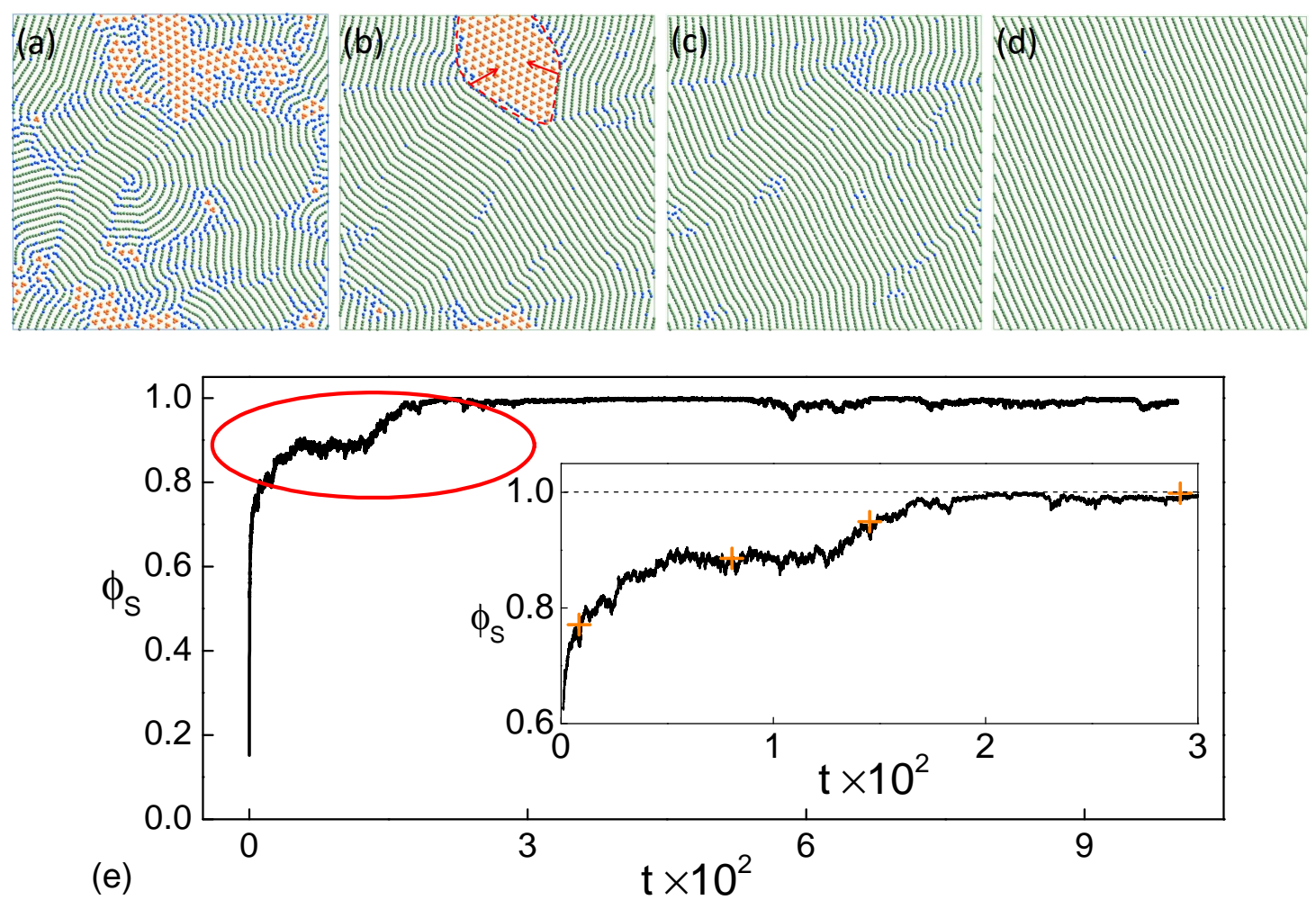

Figure 2. Formation of a highly stripe-ordered structure. (a)-(d) Representative configurations at the stage $t=10, t=90$, $t=140$ and $t=290$, respectively. The red dash line in (b) depicts a trimer domain and two red arrows point the direction that trimer domain disappears. (e) Fraction of stripe phase $\phi_{S}$ over time. Three dash lines represents the change of $\phi_{S}$ of three independent systems, and the solid line is a result over 20 times average. Inset is a zoom-in of the area in red cycle, and four moments depicted by yellow crosses correspond to (a)-(d) orderly. Note here $F_{a}=30$.

not available for the passive counterpart system. Surely the results should be dependent on the activity level $F_{a}$. In Figure 3(a)-(c), the final stationary states of the system for $F_{a}=10,40$ and 80 are presented. In contrary to the case of $F_{a}=30$ shown in Figure2(d), these patterns are not fully ordered. For a relatively small activity $F_{a}=10$, the trimer island can not be eliminated by surrounding stripe phase such that the final state is a mixture of stripe phase, trimer island and some disorder defects. For $F_{a}=30$, as we already described in Figure2(d), a highly ordered stripe structure emerges. If increasing the activity to $F_{a}=40$, however, the final state would not be that ordered, as depicted in Figure 3(b), wherein the system maintains some long range order but with many dynamic defect boundaries between ordered stripe clusters (see Movie 4). With further increasing activity to a larger value, i.e. $F_{a}=80$, the system turns into a totally disordered state as shown in Figure 3(c). Therefore, there exists an optimal level of activity (here $F_{a} \simeq 30$ ) that favorably supports the formation of ordered stripe phase. Accordingly, the stable values of $\phi_{i}(i=S, T, D)$ are depicted in Figure 3(d), wherein $\phi_{S}$ non-monotonically changes with $F_{a}$ and reaches the maximum about 1.0 for $F_{a}$ within a small range around 30 . Note that we have also studied a more larger system containing 16384 particles (see Supporting Information S2), and the same dynamic behaviors are observed.

To get more information about the final stationary states of the system, we have further investigated the dynamic and static characteristics in terms of the diffusion coefficient $\mathcal{D}$ and the radial distribution function $g(r)$, respectively. The diffusion coefficient $\mathcal{D}$ is calculated via $\mathcal{D}=\lim _{t \rightarrow \infty} \frac{1}{4 t}\left\langle\Delta r^{2}(t)\right\rangle$, where $\left\langle\Delta r^{2}(t)\right\rangle=\left\langle|\mathbf{r}(t)-\mathbf{r}(0)|^{2}\right\rangle$ is the mean square displacement (MSD) of a randomly chosen tagged particle with $\mathbf{r}(t)$ being the particle position at time $t$. The radial distribution function $g(r)$, defined as $g(r)=\frac{1}{\rho^{2}}\left\langle\sum_{i=1}^{N} \sum_{j \neq i}^{N} \delta\left(\mathbf{r}-\mathbf{r}_{i}\right) \delta\left(\mathbf{r}-\mathbf{r}_{j}\right)\right\rangle$ where $\rho=N / V$ is the number density, characterizes the probability of finding a pair of particles at a distance $r$ and thus reflects the structure of the system. In Figure $3(\mathrm{e}), \mathcal{D}$ as a function of $F_{a}$ is shown. With increasing $F_{a}, \mathcal{D}$ starts from nearly zero at the initial stage and increases sharply at $F_{a} \simeq 40$. This result indicates that stable state of the system behaves like solids for small activity $F_{a} \lesssim 40$. We note here the stable structure for $F_{a}=30$, which is very ordered, is also very stable against thermal fluctuations since the diffusion coefficient $\mathcal{D}$ is zero. If activity is large, however, the system behaves more like fluids with large $\mathcal{D}$ and loses long range order, as for example depicted in Figure 3 (c) for $F_{a}=80$. The effect of particle activity can also be reflected in $g(r)$ as drawn in Figure $3(f)$. Gen- 

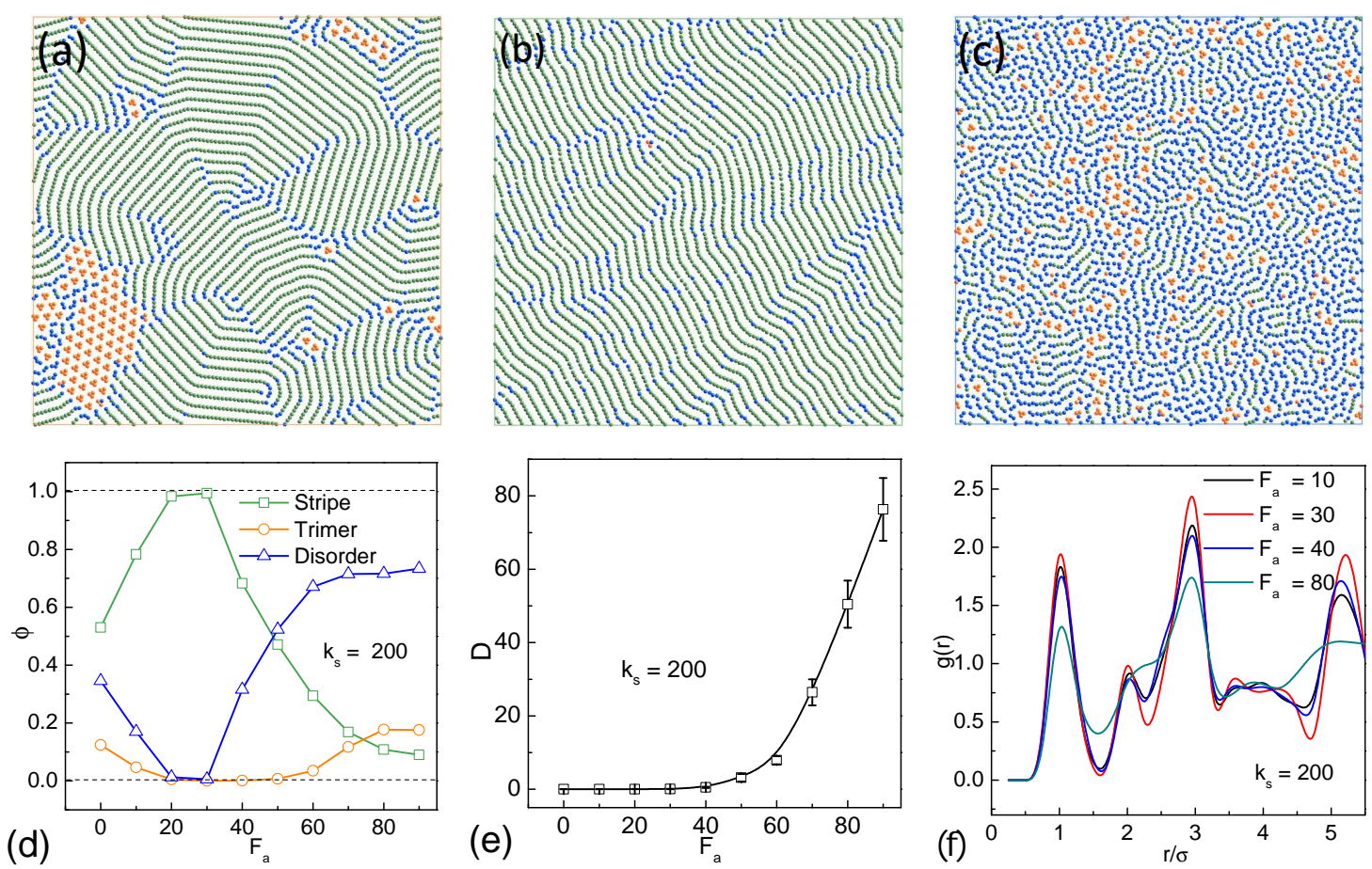

Figure 3. The impact of activity on the static and dynamic characteristics of core corona system. (a)-(c) Representative long time stable configurations at $F_{a}=10, F_{a}=40$ and $F_{a}=80$, respectively. (d) Stable values of the fractions $\phi_{i}(i=S, T, D$ corresponding to stripe, trimer and disorder, respectively) as a function of $F_{a}$. (e) Diffusion coefficient $\mathcal{D}$ as a function of $F_{a}$. (f) Radial distribution function $g(r)$ at different activities.

erally, $g(r)$ shows main peaks at $r / \sigma \simeq 1,2.7$ and 5.3 , corresponding to the typical nearest neighboring structures of the system. In particular, the peak at $r / \sigma \simeq 2.7$ corresponds to the distance between neighboring parallel stripe segments, and that at $r / \sigma \simeq 5.3$ to the distance between next-nearest parallel stripes. One can see that the peaks at $2.7 \sigma$ exist for all the activities shown, suggesting that local stripe segments maintain with the variation of $F_{a}$, and the distance between neighboring parallel segments remains nearly unchanged as can be observed for all the patterns shown in Figure/3(a)-(c) and Figure2(d). For not strong activities $F_{a}=10,30$ and 40, the peaks at $5.3 \sigma$ are remarkable, indicating the existence of some long-range order of parallel stripe patterns. The peak for $F_{a}=30$ is highest among all them, corresponding to the most ordered stripe lattice given in Figure2(d). However, for a too strong activity, say $F_{a}=80$, the peak at $5.3 \sigma$ disappears, indicating that the long-range stripe order is destroyed. All these findings clearly suggest that activity can impose two-fold effects on this core-corona system: an proper level of activity can facilitate the emergence of ordered structure, while a large one may ruin the order and make the system more disordered. Therefore, the system order shows a non-monotonic dependency on particle activity, in consistent with Figure 3(d).

The emergence of highly ordered stripe structure at an optimal level of activity (here $F_{a}=30$ ) demonstrated above, if achieved in practice, could be very promising for material design for important systems such as photonic crystal [32, 33] or template for lithography [9]. Note that this structure is stable against thermal fluctuations even if we remove the activity. To show this, we start from a defect-free state for $F_{a}=30$ and suddenly switch off the activity to $F_{a}=0$ at some time. The process is demonstrated in Figure 4(a), where the fraction of stripe phase $\phi_{S}$ is depicted as function of time. Clearly, the pattern remains unchanged and $\phi_{S}$ keeps nearly to be 1.0. More interestingly, we find that this ordered state is also "selfhealing" against strong damages. This is demonstrated in Figure 4(b), where we initialize the system in a defectfree state with $F_{a}=30$ and superpose a higher activity at some time for each particle, e.g. $F_{a}=50$, to mimic the external damaging factor and remove it at a later time. When the damage is turned on, the ordered stripe state is destroyed as expected and $\phi_{S}$ drops to a lower value. Once the damage is removed, however, $\phi_{S}$ immediately jumps back to about 1.0 and the system recovers the ordered state again (see Movie 3). Therefore, the ordered stripe state is quite stable and robust as long as the particle activity is maintained at the proper level.

The above results strongly suggests that a moderate level of activity can induce the self-assembly of highly ordered structures. Here we try to give a qualitatively understanding on this phenomenon in terms of a phenomenological free energy argument 1]. As discussed in last subsection, the equilibrium state with the lowest free 


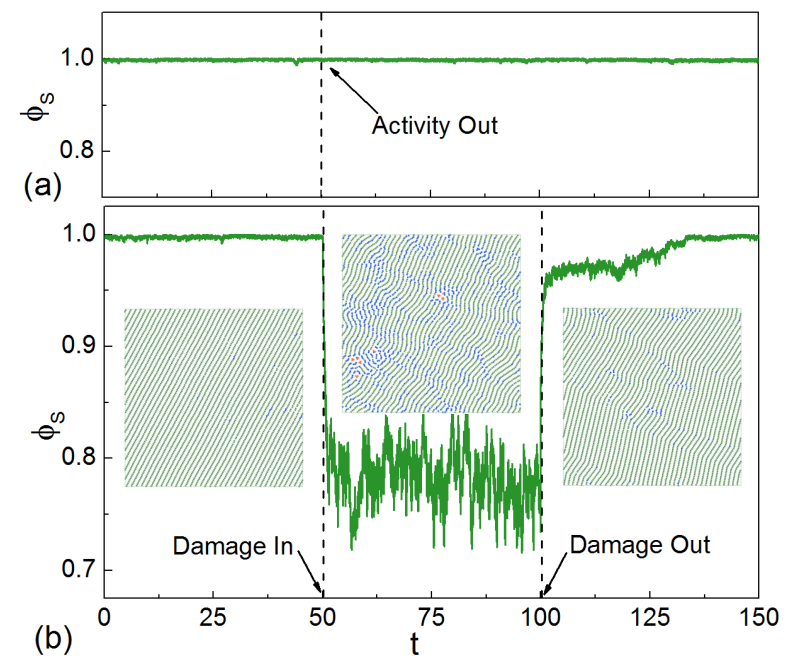

Figure 4. Stability of active induced ordered structure. (a) Against thermal fluctuations. The system is initialized in an almost perfect stripe-ordered structure $\left(\phi_{S} \simeq 1.0\right)$ with $k_{s}=$ 200 and $F_{a}=30$. And the activity is switched to zero at $t=50$ as the black arrow shown. (b) Against strong damages. The system is also initialized in $\phi_{S} \simeq 1.0$ with $k_{s}=200$ and $F_{a}=30$. At $t=50$, an activity $F_{a}=50$ modeled as the damaging factor is superposed and then it is removed at $t=100$ as the black arrows shown.

energy for $k_{s}=200$ is actually the ordered stripe phase, while that observed mixed state (shown in Figure.1(b)), with the simulation starting from a random initial condition, is a metastable state. Without activity, such a metastable state would survive for a very long time against thermal fluctuation and the ordered state cannot be reached. If particle activity is presented, however, it acts as a kind of external driving with colored noise 34. The difference of this activity from pure thermal fluctuation is that activity exerts persistence propulsion on the particle motion within the correlation time. It activity is small, the propulsion or driving force is still weak and the system still stays in the metastable state. At a proper level of activity, the system may jump out of the local minimum of free energy into the ordered state with the lowest free energy, which is what we observed for $F_{a}=30$. Since the barrier from the lowest minimum back to the metastable minimum is high, the system would stay stably in the ordered state. If activity is too strong, e.g. $F_{a}=80$, the system may jump out of the lowest minimum back to the metastable one, thus leading to disordered state again. Surely such a phenomenological description is quite qualitative and a theoretical analysis would be highly helpful, however, it is beyond the scope of current study and may deserve a separate work.

So far, we have studied the results for $k_{s}=200$, where the equilibrium state is the stripe phase. It is then interesting to investigate the phase behaviors for other values of $k_{s}$. In Figure 5(a)-(d), four representative stable patterns for $k_{s}=300$ with $F_{a}=20,60,80$ and 120 are shown, respectively. For a low activity $F_{a}=20$ in (a)
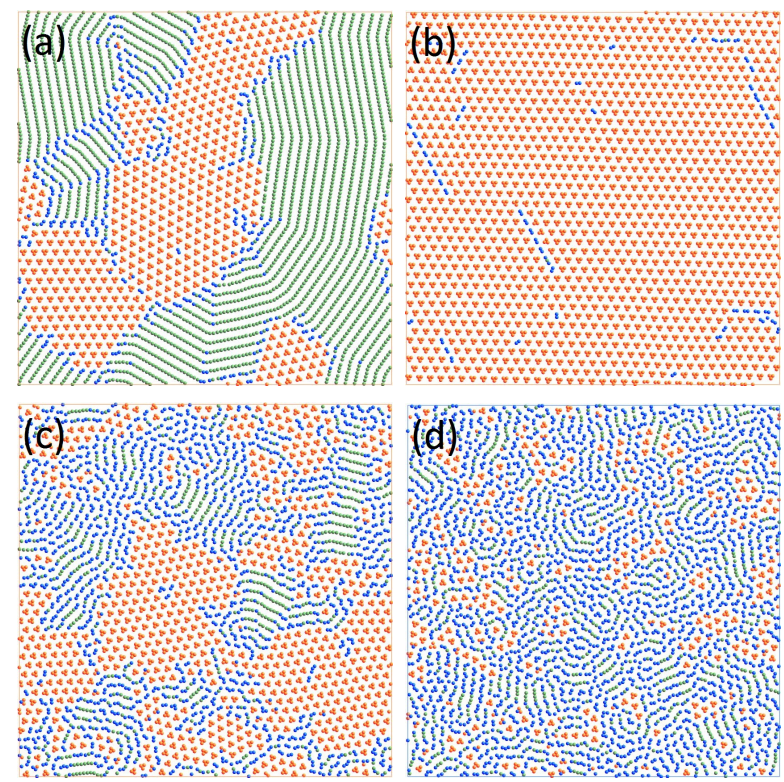

Figure 5. Representative long time stable configurations at $k_{s}=300$ for $F_{a}=20,60,80$ and 120 , corresponding to (a)(d), respectively.

, the system is now typically a mixture of trimer phase and stripe phase. For a moderate activity $F_{a}=60$ in (b), interestingly, a highly ordered trimer phase appears. The formation of such an ordered trimer phase is similar to that of the stripe phase shown in Figure 2, involving a relatively fast process forming several big clusters of trimer phases and a slow relaxation process healing the grain boundaries (see Movie 2). However, there are still many defects doped linearly inside the lattice, which is slightly different to a $k_{s}=200$ case that the system contains nearly no defects. This is due to that these defects are usually trapped as dimmers, and they could hardly be merged into trimers only when at least 3 of them meet. Note that this ordered structure also has a selfhealing feature (see Supporting Information S3). Further increasing $F_{a}$ to 80 in (c), such a trimer superlattice becomes unstable and the system contains a few clusters of trimer surrounded by disordered phase or small stripe phase. Compared to the state shown in (a), the system now is more "living" 35, i.e, the clusters may split into small pieces and then merge again (see Movie 5) while that in (a) is static. If the activity is even higher, say for $F_{a}=120$ in (d), the system behaves like liquids and becomes disorder. The non-monotonic dependency between activity $F_{a}$ and the fraction of trimer phase $\phi_{T}$ (see Supporting Information S4) is very similar to the $k_{s}=200$ case.

It is interesting to note that the activity-induced ordered structure for $k_{s}=300$ and $F_{a}=60$ is the trimer phase rather than the stripe phase, while the equilibrium state for $k_{s}=300$ is still stripe phase as suggested by Figure 1(d). This indicates that activity can not only facilitate the self-assembly process to reach the ordered 

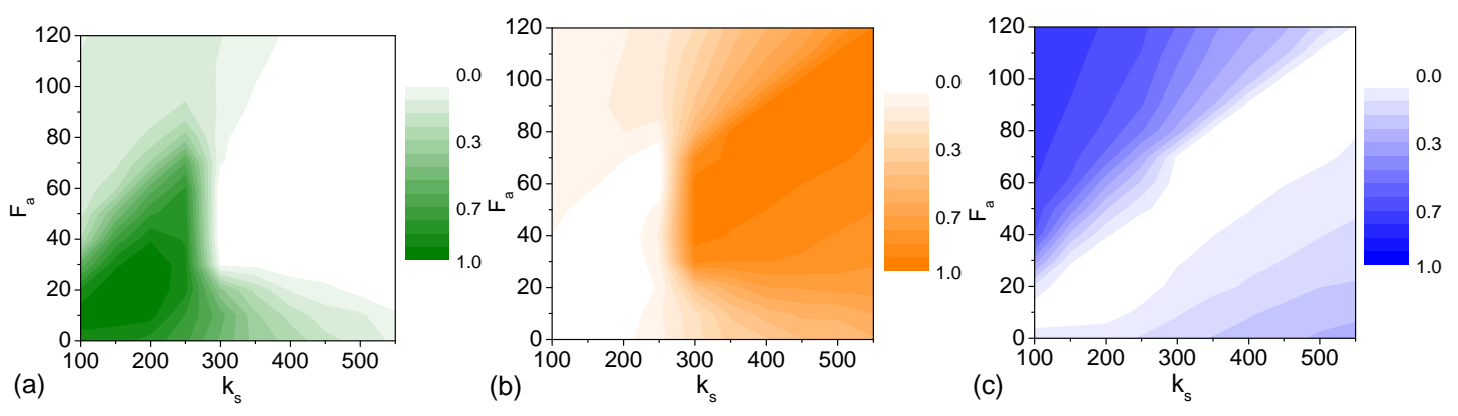

Figure 6. Non-equilibrium phase diagram of long time stable value of (a) $\phi_{S}$ colored in green, (b) $\phi_{T}$ colored in yellow and (c) $\phi_{D}$ colored in blue, respectively. The color bar attached at right-side of each phase diagram is at a range of $(0,1.0)$.

structure, but also can induce new structure that is not available in the equilibrium counterpart system. It is then very demanded to obtain a global picture by investigating the whole (nonequilibrium) phase behavior in the parameter plane spanned by $F_{a}$ and $k_{s}$. In Figure 6(a)(c), the contour plots of $\phi_{S}, \phi_{T}$ and $\phi_{D}$ in $\left(F_{a}, k_{s}\right)$ plane are presented, respectively. In (a), one can see that $\phi_{S}$ is highest in the region of small $k_{s}$ and $F_{a}$ and disappears if $k_{s}$ exceeds some threshold value. In (b), the distribution of $\phi_{T}$ is just complementary to that of $\phi_{S}$, i.e., it is highest in the region where $k_{s}$ and $F_{a}$ are large. The transition from stripe to trimer approximately takes place at $k_{s} \simeq 250$, which is not sensitive to the value of $F_{a}$ in the moderate range. Note that the transition from stripe to trimer for an equilibrium system takes place at nearly $k_{s} \simeq 650$, suggesting that activity strongly shifts the transition point. Therefore, in a broad range of $k_{s} \in(250,650)$, activity can induce a transformation from stripe to trimer. For a given $k_{s}$, both $\phi_{S}$ and $\phi_{T}$ show non-monotonic dependencies on $F_{a}$, as can be seen from these two figures. In (c), it is shown that the system is quite disordered in the left-up and right-bottom corners of the parameter plane. In the diagonal region, $\phi_{D}$ is zero, indicating that the system is in purely ordered state without defects. The optimal value of $F_{a}$ seems increases linearly with $k_{s}$ as suggested by the contour plot.

\section{CONCLUSION}

In summary, we have theoretically studied the selfassembly process of active core corona particles by using two-dimensional overdamped Langevin dynamics simulation. This system with typically two-length scales owns the possibility to form various anisotropic and super lattice structures. However, such a system would generally be trapped into a metastable state with many defects and the transition from metastable state to a thermodynamically favored one involves many local structural rearrangements, which makes the emergence of target ordered structure occur at a unreachable long time scale.
However, if exerting a moderate active force to each particle, such a metastable state can successfully cross the energy barrier leading to a large-scaled and highly ordered stripe or trimer lattice. In addition, these active induced ordered structures are stable against thermal fluctuations even when the activity is removed and can spontaneously heal strong damages as long as the activity keeps at a proper level. Our results demonstrate clearly that activity can be used as a new design strategy for self-assembly process.

It would be interesting to test our model in experimental systems, for instance, dense system of Janus particles coated by microgels in alternating electric field. The particles could exhibit spontaneous motility in such field[36, 37] and repel each other due to the ambient soft microgels [15. In practice, spring potential may not exactly hold when two coronas are strongly extrude, while one can alternatively use an external field to achieve a more robust repulsive interaction. For example, N. Osterman et.al used an external magnetic field to achieve the repulsion between superparamagnetic spheres which are trapped in a thin wedge-shaped cell, and softened the repulsion by changing the cell thickness [27. Since the two key ingredients, corona[13, 15, 28] and activity [23, 25, 38, 40, are ubiquitous in many real systems and widely studied both theoretically and experimentally, we hope that our results could open new perspectives to the fabrication of similar highly ordered and self-healing structure and new insights to defect engineering in material field.

\section{ACKNOWLEDGMENTS}

This work is supported by MOST(2016YFA0400904, 2018YFA0208702), by NSFC (21833007, 21790350, 21673212, 21521001, 21473165, 21403204), by the Fundamental Research Funds for the Central Universities (2340000074), and Anhui Initiative in Quantum Information Technologies (AHY090200). 
[1] M. A. Boles, M. Engel, and D. V. Talapin, Chemical reviews 116, 11220 (2016).

[2] C. A. S. Batista, R. G. Larson, and N. A. Kotov, Science 350, 1242477 (2015).

[3] M. A. Boles and D. V. Talapin, Journal of the American Chemical Society 137, 4494 (2015).

[4] X. Ye, C. Zhu, P. Ercius, S. N. Raja, B. He, M. R. Jones, M. R. Hauwiller, Y. Liu, T. Xu, and A. P. Alivisatos, Nature communications 6, 10052 (2015).

[5] J. Hou, M. Li, and Y. Song, Angewandte Chemie International Edition 57, 2544 (2018).

[6] L. Sun, H. Lin, K. L. Kohlstedt, G. C. Schatz, and C. A. Mirkin, Proceedings of the National Academy of Sciences 115, 7242 (2018).

[7] F. Li, X. Huang, J. Lu, J. Ma, and Z. Liu, Nature Physics 14, 30 (2018).

[8] H. He, C. Qiu, L. Ye, X. Cai, X. Fan, M. Ke, F. Zhang, and Z. Liu, Nature 560, 61 (2018).

[9] B. M. Rey, R. Elnathan, R. Ditcovski, K. Geisel, M. Zanini, M.-A. Fernandez-Rodriguez, V. V. Naik, A. Frutiger, W. Richtering, T. Ellenbogen, et al., Nano letters 16, 157 (2015).

[10] K. J. Si, Y. Chen, Q. Shi, and W. Cheng, Advanced Science 5, 1700179 (2018).

[11] G. Malescio and G. Pellicane, Nature materials 2, 97 (2003).

[12] E. Jagla, Physical Review E 58, 1478 (1998).

[13] T. Dotera, T. Oshiro, and P. Ziherl, Nature 506, 208 (2014).

[14] R. Ruiz, H. Kang, F. A. Detcheverry, E. Dobisz, D. S. Kercher, T. R. Albrecht, J. J. de Pablo, and P. F. Nealey, Science 321, 936 (2008).

[15] M. Rey, A. D. Law, D. M. A. Buzza, and N. Vogel, Journal of the American Chemical Society 139, 17464 (2017).

[16] M. I. Bodnarchuk, E. V. Shevchenko, and D. V. Talapin, Journal of the American Chemical Society 133, 20837 (2011).

[17] A. Aubret, M. Youssef, S. Sacanna, and J. Palacci, Nat. Phys 14, 1114 (2018).

[18] Y. Fily and M. C. Marchetti, Physical review letters 108, 235702 (2012).

[19] I. Buttinoni, J. Bialké, F. Kümmel, H. Löwen, C. Bechinger, and T. Speck, Physical review letters 110, 238301 (2013).

[20] J. Palacci, S. Sacanna, A. P. Steinberg, D. J. Pine, and P. M. Chaikin, Science, 1230020 (2013).
[21] J. Dunkel, S. Heidenreich, K. Drescher, H. H. Wensink, M. Bär, and R. E. Goldstein, Physical review letters 110, 228102 (2013).

[22] J. A. Cohen and R. Golestanian, Physical review letters 112, 068302 (2014).

[23] S. A. Mallory, C. Valeriani, and A. Cacciuto, Annual review of physical chemistry 69, 59 (2018).

[24] S. Mallory and A. Cacciuto, Physical Review E 94, 022607 (2016).

[25] S. A. Mallory and A. Cacciuto, Journal of the American Chemical Society (2019).

[26] W. Cheng, M. R. Hartman, D.-M. Smilgies, R. Long, M. J. Campolongo, R. Li, K. Sekar, C.-Y. Hui, and D. Luo, Angewandte Chemie International Edition 49, $380(2010)$.

[27] N. Osterman, D. Babič, I. Poberaj, J. Dobnikar, and P. Ziherl, Physical review letters 99, 248301 (2007).

[28] M. Zu, P. Tan, and N. Xu, Nature communications 8, 2089 (2017).

[29] H. G. Schoberth, H. Emmerich, M. Holzinger, M. Dulle, S. Förster, and T. Gruhn, Soft matter 12, 7644 (2016).

[30] K. Barkan, M. Engel, and R. Lifshitz, Physical review letters 113, 098304 (2014).

[31] G. Malescio and G. Pellicane, Physical Review E 70, 021202 (2004).

[32] C. Park, K. Koh, and U. Jeong, Scientific reports 5, 8340 (2015).

[33] M. Kolle, P. M. Salgard-Cunha, M. R. Scherer, F. Huang, P. Vukusic, S. Mahajan, J. J. Baumberg, and U. Steiner, Nature nanotechnology 5, 511 (2010).

[34] É. Fodor, C. Nardini, M. E. Cates, J. Tailleur, P. Visco, and F. van Wijland, Physical review letters 117, 038103 (2016).

[35] N. H. Nguyen, D. Klotsa, M. Engel, and S. C. Glotzer, Physical review letters 112, 075701 (2014).

[36] J. Yan, M. Han, J. Zhang, C. Xu, E. Luijten, and S. Granick, Nature materials 15, 1095 (2016).

[37] A. Bricard, J.-B. Caussin, N. Desreumaux, O. Dauchot, and D. Bartolo, Nature 503, 95 (2013).

[38] A. E. Patteson, A. Gopinath, and P. E. Arratia, Nature communications 9, 5373 (2018).

[39] D. Geyer, A. Morin, and D. Bartolo, Nature materials 17, 789 (2018).

[40] K.-T. Wu, J. B. Hishamunda, D. T. Chen, S. J. DeCamp, Y.-W. Chang, A. Fernández-Nieves, S. Fraden, and Z. Dogic, Science 355, eaal1979 (2017). 